\title{
Erratum to: Helicocentralis hyalina gen. et sp. nov., an aero-aquatic helicosporous fungus (Leotiomycetes, Ascomycota) in Thailand
}

\author{
Veera Sri-indrasutdhi ${ }^{1}$ - Clement K. M. Tsui ${ }^{2} \cdot$ Charuwan Chuaseeharonnachai $^{1}$. \\ Kaoru Yamaguchi ${ }^{3}$ - Satinee Suetrong ${ }^{1}$ - Izumi Okane ${ }^{4}$. Akira Nakagiri ${ }^{5}$. \\ Nattawut Boonyuen ${ }^{1}$
}

Published online: 13 October 2015

(C) German Mycological Society and Springer-Verlag Berlin Heidelberg 2015

\section{Erratum to: Mycol Progress (2015) 14:81}

DOI 10.1007/s11557-015-1103-6

In the original version of this article Fig. 3 was inadvertently published twice, and Fig. 2 is missing.

The corrected figures are as follows:

The authors regret this mistake.

The online version of the original article can be found at http://dx.doi.org/ 10.1007/s11557-015-1103-6.

Veera Sri-indrasutdhi

Veera@biotec.or.th

1 Fungal Biodiversity Laboratory, BIOTEC, National Science and Technology Development Agency (NSTDA), 113 Thailand Science Park, Thanon Phahonyothin, Tambon Khlong Nueng, Amphoe Khlong Luang, Pathum Thani 12120, Thailand

2 Department of Pathology and Laboratory Medicine, University of British Columbia, Vancouver, BC V6T 1Z4, Canada

3 Biological Resource Center (NBRC), National Institute of Technology and Evaluation (NITE), 2-5-8 Kazusakamatari, Kisarazu, Chiba 292-0818, Japan

4 Faculty of Life and Environmental Sciences, University of Tsukuba, 1-1-1 Tennodai, Tsukuba, Ibaraki 305-8572, Japan

5 Fungus/Mushroom Resource and Research Center, Faculty of Agriculture, Tottori University, 4-101 Koyama-Minami, Tottori, Tottori 680-8553, Japan 
Fig. 2 One of seven MPTs inferred from combined analyses of nuc18S and nuc28S rDNA sequences. BSMP, BSML bootstrap support values $\geq 50 \%$ and BYPPs $\geq 0.95$ are given at left above the node, right above the node and below the node, respectively. The original code numbers of these sequences selected for phylogenetic analyses are given in the tree after the taxon names

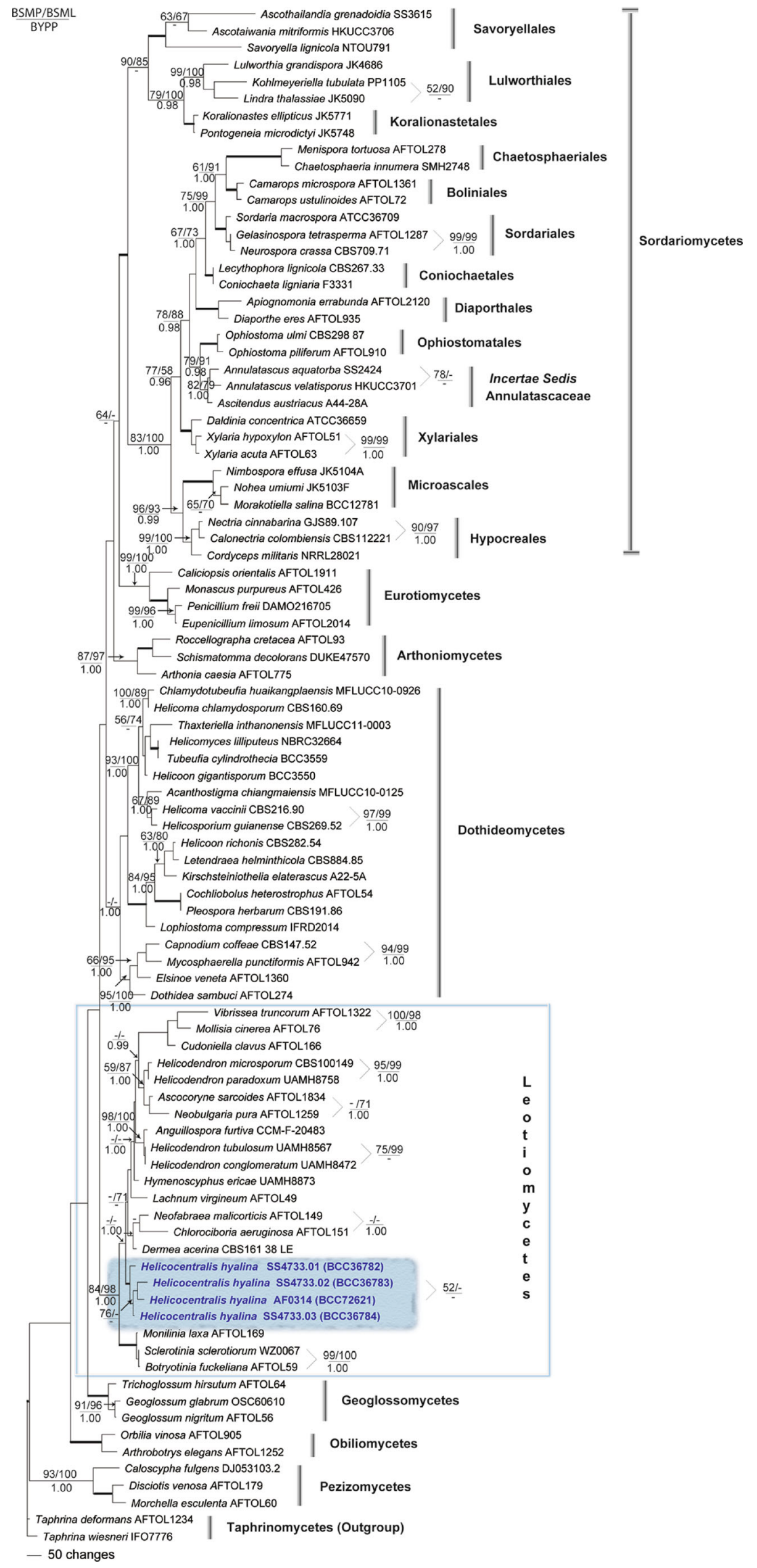


Fig. 3 The best MPT obtained from the ITS rDNA dataset. Bootstrap resampling values $\geq 50$ $\%$ are given above the branches, and BYPPs $\geq 0.95$ are indicated below the nodes. The original code numbers of these sequences selected for phylogenetic analyses are given in the tree after the taxon names
$69 \%$

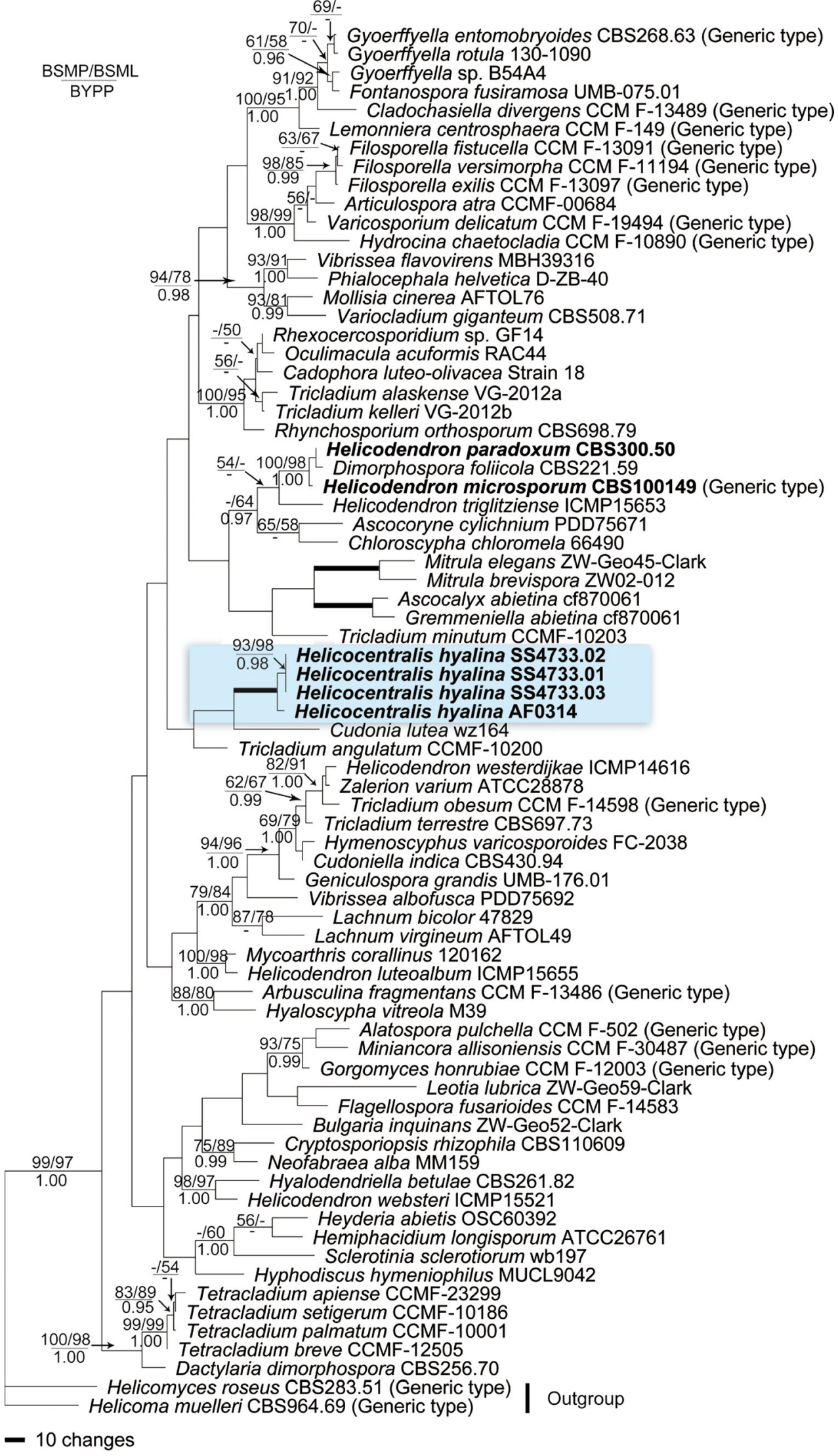

